\title{
Investigating the relationship between active learning task characteristics and student success
}

\author{
Leanne Doughty, ${ }^{1}$ Laurel Hartley, ${ }^{2}$ Paul Le, ${ }^{2}$ Mary Nyaema, ${ }^{3}$ Jeffrey Boyer, ${ }^{4}$ and Robert M. Talbot III ${ }^{1}$ \\ ${ }^{1}$ School of Education and Human Development, University of Colorado Denver, 1380 Lawrence St, Denver, Colorado, 80204 \\ ${ }^{2}$ Department of Integrative Biology, University of Colorado Denver, 1151 Arapahoe St, Denver, Colorado, 80204 \\ ${ }^{3}$ STEM Transformation Institute, Florida International University, 11200 SW 8th St, Miami, Florida, 33199 \\ ${ }^{4}$ College of Science and Mathematics, North Dakota State University, \\ 1340 Administration Ave, Fargo, North Dakota, 58105
}

\begin{abstract}
There is strong evidence that the implementation of active learning in undergraduate science courses can lead to increased student conceptual understanding and course achievement, but we still do not know what specific characteristics of active learning contribute the most to student success. Our work examines the tasks that students are asked to engage with during active learning, with the goal of investigating the relationship between different task characteristics and student level outcomes. To this end, we are working to characterize the active learning tasks that students engage with in the classroom with respect to authenticity and cognitive depth. This paper presents our characterization of the tasks we have collected from four introductory physics courses at three institutions and discusses the relationship between these characteristics and student gains on the Force and Motion Conceptual Evaluation instrument.
\end{abstract}

\section{INTRODUCTION}

The use of active learning methods is increasingly widespread in undergraduate science classrooms. There is strong evidence that the implementation of active learning methods can lead to better student outcomes like increased conceptual understanding and course achievement [1]. Definitions of active learning are broad and include students being engaged in activities and discussions as opposed to passively listening in more traditional teaching approaches [2]. The term covers a range of intersecting activity formats (e.g. problem solving, experimentation) and social interaction types (e.g. individual, peers) [2]. Different reform goals have led to the development of different methods, and these differing goals along with failed communications during dissemination have resulted in differentiated implementations [3]. Not all of these varying methods and implementations contribute equally to student level outcomes [4]. Our work is concerned with determining specific aspects of active learning that contribute to student success [5].

One important element of active learning is the tasks with which students are asked to engage. The study presented in this paper investigates the relationship between active learning task characteristics and student success. Our hypothesis is that students will be more successful in courses where they are engaged in authentic group-worthy tasks. Section II outlines our theoretical framework which motivates our examination of active learning tasks and guides our characterization. Section III describes our data collected in four introductory mechanics courses at three different institiutions. In Section IV we provide details of our analysis of those tasks and we present and discuss our results in Section V.

\section{THEORETICAL FRAMEWORK}

In our work, we use Activity Theory [6] to frame and conceptualize the teaching and learning of science in the under- graduate science classroom. This framework allows you to consider an individual sudent's learning as measured by outcomes of interest (e.g. course grade, learning gains on an assessment) through the subject-object-outcome space, while the other vertices encorporate the mediational factors that are present in a complex classroom environment (see Fig. 1).

In taking this approach for our work, we are interested in the Activity of Learning Science. In the Learning Science System, there are many actors in the system that make up the Community (students, instructors, learning assistants, etc). The primary purpose of the system is to support student learning, therefore we center the student in this model. In our system the mediating artifacts are defined as all of the materials and resources that members of the classroom community engage with or around. These include textbooks, lecture slides, worksheets, etc. We operationalize the rules of the system as the norms of thinking and doing in the classroom, i.e. the implicit and explicit guidelines for the way members of the classroom community should behave. The division of labour in our system is defined as the responsibilities of each member of the community. These responsibilities are visible through the actions each member makes to enact the classroom norms. For example, in an active learning classroom, students may be responsible for building their own understanding of class concepts by interacting with other students, interacting with active learning tasks, asking questions, etc. The instructor and LAs may be responsible for facilitating by designing active learning tasks, encouraging students to work together, guiding student group discussions, etc.

While these components of the activity system are dynamic and interactional in nature, it is neccessary to first focus on components individually in order to make sense of the system as a whole. Here, we take a closer look at the the mediational artifacts, specifically the active learning tasks used in the classroom. From a situated cognition perspective [7] knowledge is a product of activity, context, and culture, and as such learning takes place through a cognitive apprenticeship. From this, we hypothesize that successful learning is more 

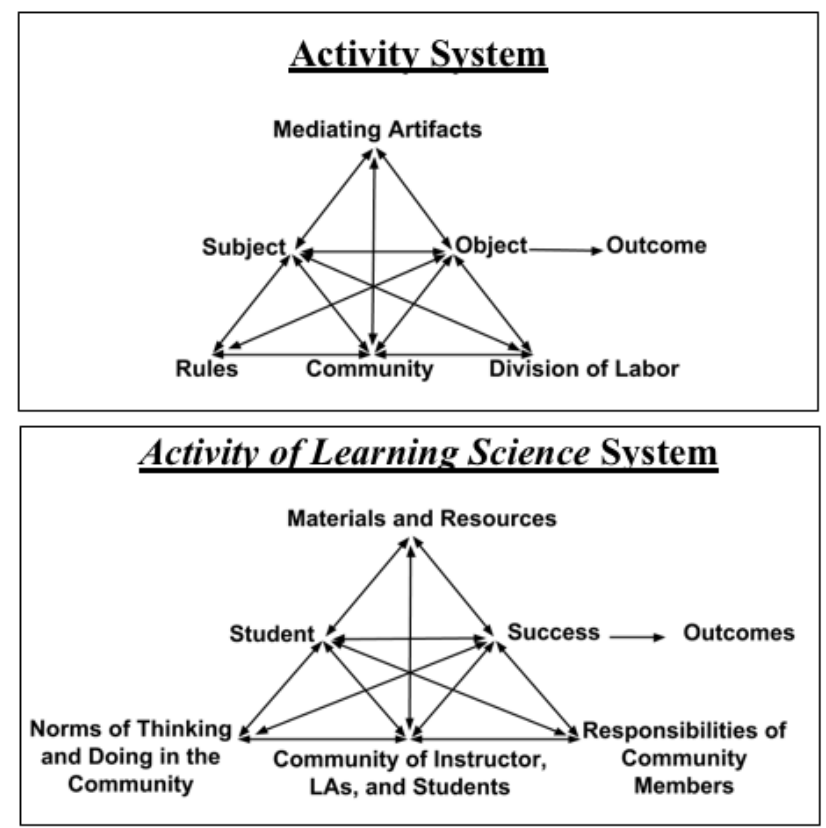

FIG. 1: The Activity system as definied by Engestrom [6] (above) and our operationalized system for the Activity of Learning Science (below).

likely to occur when students are given opportunities to do activities that scientists engage in, in ways that scientists do (i.e. authentic tasks solved through social interaction). Also assuming that students will be more likely to interact around tasks that are more cognitively complex, we will characterize learning tasks in terms of authenticity and cognitive complexity. The tools used to make those characterizations are described in more detail in Section IV.

\section{DATA}

The data presented in this paper comes from four different introductory mechanics courses at three institutions (see Table I). The class sizes for all four courses were relatively small ranging from 42 to 73 students. The classroom set-up for Course A was traditional with movable chairs that faced towards the front of the room, while the classrooms for the other three courses were studio-style with students in grouped tables at all times. The amount of active learning that occured in each course varied from an average of $34 \%$ of class time for Course A to and average of $69 \%$ of class time for Course D (based on the Ind, CG, WG and OG COPUS student codes).

Tasks were collected during classroom observations where the Classroom Observation Protocol for Undergraduate STEM (COPUS) [8] was being used to identify types of active learning methods. Anything students were working on during the Ind (individual thinking/problem solving), CG (discussing clicker questions), WG (working in groups on
TABLE I: Characteristics of our four sampled introductory mechanics courses, and details of task and concept inventory data collected in each course.

\begin{tabular}{|l|cccc|}
\hline & Course A & Course B & Course C & Course D \\
\hline Class size & 42 & 44 & 73 & 73 \\
Classroom & Traditional & Studio & Studio & Studio \\
$\%$ active & $34 \%$ & $59 \%$ & $50 \%$ & $69 \%$ \\
\hline Observations & 7 & 13 & 16 & 10 \\
Tasks/day & 5 & 6 & 5 & 2 \\
Length (mins) & 7 & 6 & 7 & 46 \\
\hline CI pre score $(\bar{x})$ & $20.3 \%$ & $36.7 \%$ & $32.3 \%$ & $18.4 \%$ \\
CI post score $(\bar{x})$ & $37.5 \%$ & $62.2 \%$ & $64.7 \%$ & $39 \%$ \\
CI gain $(d)$ & 0.91 & 0.95 & 1.41 & 1.30 \\
\hline
\end{tabular}

a worksheet activity) and OG (other assigned group activity) codes was classified as a task. The collection method varied by task type. For example, physical copies of worksheets were gathered, photos were taken of questions posed on slides, and questions posed verbally by the instructor were recorded. Courses $\mathrm{A}, \mathrm{B}$ and $\mathrm{C}$ had a similar number of tasks per day (5-6) and a similar average task length (6-7 minutes). Course D had much fewer tasks that lasted almost 40 minutes more on average. The task types used in courses B and C consisted on mostly Peer Instruction [9] style clicker questions and guided-inquiry style worksheets. Course A used textbook style problems and clicker questions. Course D tasks usually involved hands-on experiments. In all courses, activities were facilitated by the instructor and Learning Assistants [10] who walked around the room and interected with individual students and student groups.

Our student success outcome was measured through student learning gains on the The Force and Motion Conceptual Evaluation (FMCE) [11]. We acknowledge that operationalizing student success through learning gains takes a narrow perspective on what it may mean to be successful in a course. However, it is typically the way in which education researchers compare the impact of different teaching and learning strategies and interventions [12]. Using concept inventories over course grade is advantageous in that they are research-based, validated, and standarized [12]. We used Cohen's $d$ [13] as our measure of student learning gains. This value and the average pre and post test scores for each course are shown in Table I. Cohen's $d$ takes into account the standard deviation for each score distribution. While courses A and D have similar average pre and post scores (as do courses $\mathrm{B}$ and $\mathrm{C}$ ), the learning gains are different in each case beacuse the spread in scores are different.

\section{ANALYSIS}

The tasks were analyzed using two tools. The ThreeDimensional Learning Assessment Protocol (3D-LAP) [14] 


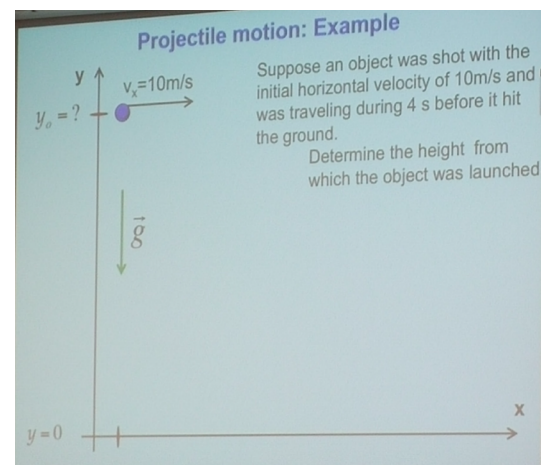

(a) Sample Task A

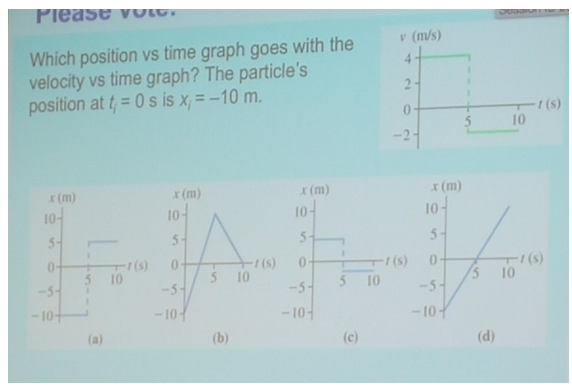

(b) Sample Task B

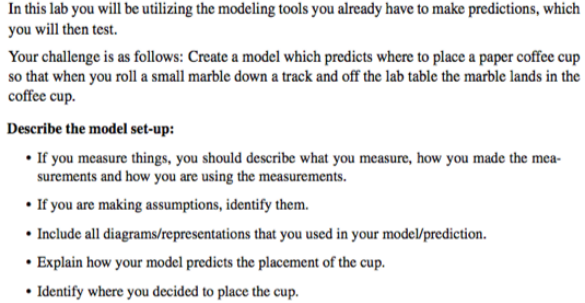

(c) Sample Task C

FIG. 2: Sample tasks collected from the introductory mechanics courses.

provided us with a measure of task authenticity. The 3DLAP is based on the idea of three-dimension learning [15] which promotes the integration of scientific practices (e.g. developing and using models, and using computational and mathematical thinking), ways of thinking that span the science disciplines (e.g. systems and system models, proportion and quantity), and disciplinary core ideas in developing tasks (e.g. interactions cause changes in motion). Not only does engaging in scientific practice align with the way we described authenticity, but also focusing on a few central concepts creates further alignment because it is around those that science practitioners organize and contextualize their knowledge [16]. The 3D-LAP outlines criteria that a task must meet in order to determine if that task is likely to prompt student enagement with any of the three dimensions. We used the revised Bloom's Taxonomy [17] to determine the cognitive complexity of a task. The taxonomy describes six hierarchical cognitive processes by which learners engage with knowledge: remember, understand, apply, analyze, evaluate, create. Figure 2 shows some sample tasks and Table II shows the correspoding characterization for those tasks. One coder coded all of the tasks for all four courses. Another coder coded a random $25 \%$ of tasks for each course. The \% agreement was 99\% for Bloom's level and 96\% for the 3D-LAP codes.

TABLE II: Bloom's Level and 3D-LAP characterizations for sample tasks shown in Figure 2.

\begin{tabular}{l|ccc}
\hline \hline & Task A & Task B & Task C \\
\hline Bloom's Level & Apply & Understand & Create \\
\hline Core Idea & $\begin{array}{c}\text { Interactions } \\
\text { cause changes } \\
\text { in motion }\end{array}$ & $\begin{array}{c}\text { Interactions } \\
\text { cause changes } \\
\text { in motion }\end{array}$ & $\begin{array}{c}\text { Interactions } \\
\text { cause changes } \\
\text { in motion }\end{array}$ \\
$\begin{array}{l}\text { Crosscutting } \\
\text { Concept }\end{array}$ & None & $\begin{array}{c}\text { Proportion } \\
\text { and quantity }\end{array}$ & None \\
\hline $\begin{array}{l}\text { Scientifc } \\
\text { Practice }\end{array}$ & None & None & $\begin{array}{c}\text { Developing } \\
\text { and using models }\end{array}$ \\
\hline \hline
\end{tabular}

\section{RESULTS AND DISCUSSION}

The 3D-LAP rating for tasks from all four courses is summarized in Figure 3a in terms of the number of dimensions present per task. The majority of tasks in all courses were one-dimensional containing just a core idea (Interactions Can Cause Changes in Motion, Energy is Conserved, or Exchanges of Energy increase Total Entropy). Only courses C and $\mathrm{D}$ had any three-dimensional tasks. Overall, the vast majority of tasks could not be considered authentic with courses $\mathrm{A}, \mathrm{B}$ and C only having $11 \%, 3 \%$ and $13 \%$ of their tasks containing scientific practices. Course D had the most authentic tasks with $31 \%$ of them being three-dimensional and $41 \%$ containg scientific practices. The cross-cutting concepts in the tasks across all four courses were Proportion and Quantity and Systems and System Models. The scientific practices present were Analyzing and Interpreting Data, Developing and Using Models, Using Mathematics and Computational Thinking, and Constructing Explanations and Engaging in Argument from Evidence.

The distribution of tasks across the Bloom's levels in all four courses is shown in Figure 3b. For all four courses, the majority of tasks are in the Understand and Apply levels. Only course D has tasks (25\%) above the Apply level. Despite barely any tasks being at the lowest level, overall the majority of tasks were still not very cognitively complex.

So what is the relationship between the task characteristics of authenticity and cognitive complexity and student success as measured by effect size of FMCE scores? There is some indication of a positive correlation between task authenicty and student success. Courses C and D are the only two courses that had three-dimensional tasks and those two courses has the highest effect sizes, 1.41 and 1.30 respectively. Contradiciting this however, course D had more three-dimensional tasks and yet had slightly lower effect size than course C. With regard to cognitive complexity, this is a similar story for course D. Course B's tasks had the lowest Bloom's rating being the only course to have some tasks at the Remember level and having more Understand and less Apply than 


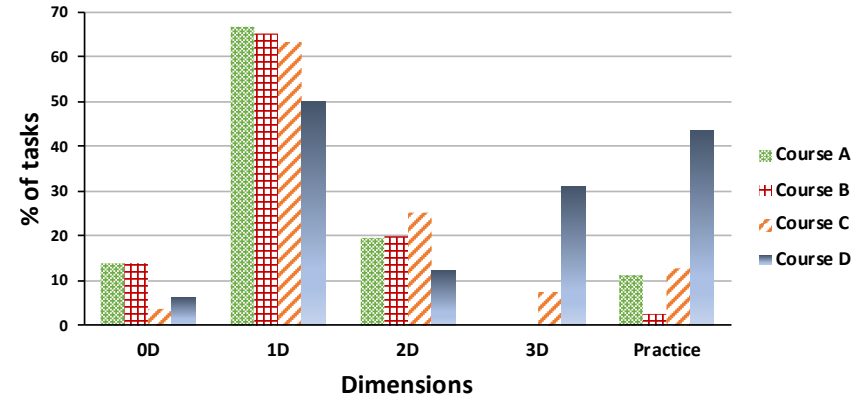

(a) 3D-LAP rating for tasks in each course.

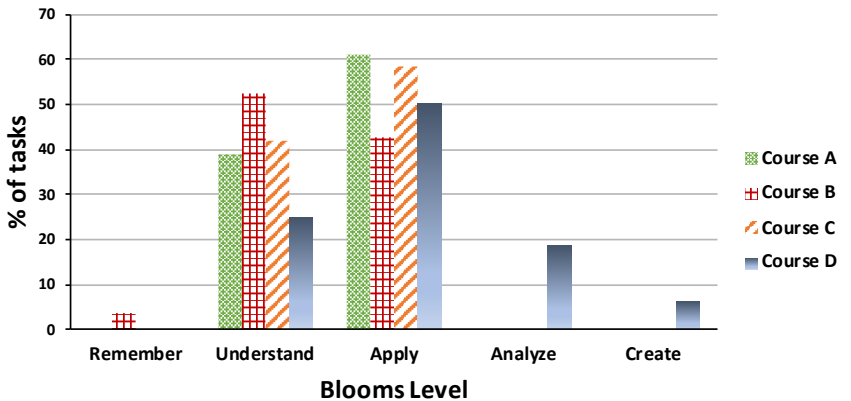

(b) Bloom's Level rating for tasks in each course.

FIG. 3: Summary of 3D-LAP and Blooms' Level rating for tasks in each course.

course A, yet had a comparable effect size to course A. Given the number of courses that we have sampled thus far, we are not able to make a claim about the relationship between task characteristics and student success in terms of statistical significance. However, we are currently extending this study to more introductory physics, chemistry, and biology courses and will be able to investigate the strength of the correlation.

Interpreting the active-learning task-student success relationship is complex. As we discussed in our theoretical framework section the mediating artifacts are part of a system. While focusing on characterizing active learning tasks is valuable in helping make sense of one part of the classroom system there are many other factors that will influence how effectively those artifacts can mediate the learning of science. If the norms of the classroom and the responsibilities of the actors in the classroom community are different, likely seen in the implementation of the active learning tasks, then activity theory suggests that these differences will impact the effect that a given mediational artifact can have on student success. The goal of our larger work is to look at the interactions between the characteristics of the mediational artifacts and other components of our Activity of Learning Science system and how they connect to impact student success.

\section{ACKNOWLEDGMENTS}

We thank all the members of our research team at University of Colorado Denver, Florida International University, and North Dakota State University who aided with the data collection and provided valuable feedback on the analysis. This work is supported by NSF DUE award 1525115.
[1] S. Freeman, S. L. Eddy, M. McDonough, M. K. Smith, N. Okoroafor, H. Jordt, and M. P. Wenderoth, PNAS 111, 23 (2014)

[2] A. K. Wood, R. Galloway, R. Donnelly, and J. Hardy, Phys. Rev. Phys. Educ. Res. 12, 010140 (2016)

[3] M. Dancy and C. Henderson, Phys. Rev. Phys. Educ. Res. 3, 010103 (2007)

[4] M. A. Ruiz-Primo, D. Briggs, H. Iverson, R. Talbot, L. A. Shepard, Science. 331, 6022 (2011)

[5] R. Talbot et al., in PERC 2016 Proceedings, (Sacramento, Californai, 2016)

[6] Y. Engestrom, Learning by Expanding: An Activity-theoretical Approach to Developmental Research (Orienta-Konsultit Oy, 1987)

[7] J. S. Brown, A. Collins and P. Duguid, Educational Researcher. 18, 32 (1989)

[8] M. K. Smith, F. H. M. Jones, S. L. Gilbert, and C. E. Wieman, CBE Life Sci. Educ. 12, 618 (2013)
[9] E. Mazur, Peer Instruction: A User's Manual (2013)

[10] V. Otero, N. Finkelstein, R. McCray, and S. J. Pollock, Science 313, 445 (2006)

[11] R. Thornton and D. Sokoloff, Am. J. Phys. 66, 4 (1998)

[12] J. M. Nissen, R. M. Talbot, A. N. Thompson and B. Van Dusen, Phys. Rev. Phys. Educ. Res. 14, 010115 (2018)

[13] J. Cohen, Statistical Power Analysis for the Beahavioral Sciences (NY, 1969)

[14] J. T. Laverty et al., PloS one, 11, 9 (2016)

[15] National Research Council, A framework for $K-12$ science: Practices, crosscutting concepts, and core ideas. (Nat. Acad. Press, 2012)

[16] J. D. Bransford, A. L. Brown and R. R. Cocking, How people learn: Brain, mind, experience, and school. (Nat. Acad. Press, 1999)

[17] L. W. Anderson et al, A taxonomy for learning, teaching, and assessing: A revision of Bloom's taxonomy of educational objectives (NY, 2001) 